\title{
Giant ethmoido-frontal osteoma with intracranial and orbital extension - Case report
}

\author{
Vlad Budu',2, Tatiana Decuseara', Andreea Nicoleta Costache', Loredana Ghiuzan', \\ Lavinia Sava', Monica Hodor ${ }^{3}$, Vadim Palii ${ }^{3}$, Mihai Tusaliu' ${ }^{1,2}$ \\ 1"Prof. Dr. D. Hociota" Institute of Phono-Audiology and Functional Surgery, Bucharest, Romania \\ 2"Carol Davila" University of Medicine and Pharmacy, Faculty of Medicine, Bucharest, Romania \\ ${ }^{3}$ Floreasca Emergency Clinical Hospital, Bucharest, Romania
}

\begin{abstract}
BACKGROUND. The most frequent benign nasal tumor is the osteoma. It is commonly asymptomatic and usually an incidental finding and can be caused by multiple factors. The therapeutic approach for symptomatic osteomas is strictly surgical.

CASE REPORT. The authors present a patient with giant ethmoido-frontal osteoma, causing the compression of the ocular globe and the right frontal lobe. Considering the intracranial extension, a combined surgical team consisting of an ENT surgeon and neurosurgeon decided to deal with the case. The tumoral mass was removed with no recurrence after a follow-up of two years.
\end{abstract}

CONCLUSION. Surgery via external and endoscopic approach aims to completely resect the osteoma with frontal sinus origin and orbital or intracranial extension.

KEYWORDS: frontal osteoma, endoscopic sinus surgery, orbital complications.

\section{INTRODUCTION}

Osteoma is the most frequent tumor of the nose and paranasal sinuses, with a predominant occurrence in the frontal sinus ${ }^{1}$. It is a slow-growing tumor and it can be associated with a sinus mucocele or chronic rhinosinusitis due to an inefficient drainage of the frontal sinus. The initial symptom, mainly determined by sinus drainage obstruction, includes pain and tenderness in the craniofacial region ${ }^{2}$. A significant growth of the tumor can determine ocular complications such as diplopia, proptosis, limitation of eye movement and intracranial complications, such as meningitis, subdural or cerebral abscess. The presence of symptoms is a firm indication for surgical management.

The case history, clinic examination, nasal endoscopy and computed tomography are the required methods for the diagnosis protocol of sinonasal osteomas.

The clinical presentation varies depending on the size and location of the tumor. For the frontal sinus osteoma the symptoms include facial pressure, headaches with no response to usual treatment, mucopurulent discharge, even facial or frontal asymmetry, exophthalmos, diplopia, palpebral edema. In addition, the patient can present behavioural disorder or intracranial hypertension.

The authors report a giant right ethmoido-frontal osteoma with right orbital extension and endocranial involvement. The tumor was bulging into the right subfrontal region and, despite the bony destructions of the orbital walls and the skull base, the patient presented minimal symptoms.

\section{CASE REPORT}

A 25-year-old woman was admitted to our clinic with a right frontal and ethmoidal tumor with a progressive evolution in the last ten months. She presented normal eye movement and sight, despite the minimal lateral displacement and anterior proptosis of the right eye. The clinical ENT examination revealed an immovable tumor in the inner angle of the right eye. It had a firm, plain consistency, ellipsoidal shape, 2 × 2 $\mathrm{cm}$ size (by manual palpation) and did not cause spontaneous or induced pain.

The nasal endoscopy showed indirect signs of the tumor: hypertrophy of the agger nasi cell, frontal recess obstruction and an enlarged uncinate process, causing 
incomplete blockage of the right middle meatus.

The neurological examination revealed a conscious and cooperating patient, with 15 points on Glasgow scale, no motor or sensory deficits and no cranial nerves dysfunction.

The cerebral CT scan described a tumoral mass of bony density, having $5 \times 4.5 \times 3.5 \mathrm{~cm}$ in size, probably originating from the right frontal sinus, with inferior extension, causing destruction of the frontal sinus orbital wall, at the right and left ethmoid level. In addition, partial destruction of the right medial orbital wall was present. This space-occupying mass determined destruction of the frontal sinus posterior wall, projecting into the dura mater and compressing the right frontal lobe (Figure 1).

MRI scan of the head revealed that the dura mater at the frontal lobe region was intact, thus the tumor had no intracranial extension.

Following these investigations, we decided for a multidisciplinary surgical approach, ENT and neurosurgery. In this particular case of a giant frontal osteoma with both inferior extension to the orbit and the nasosinusal region and posterior extension to the frontal lobe, the surgical decision was a combined external and endoscopic technique.

Considering the complexity of the case and the possible complications, the surgery was performed at the Neurosurgery Clinic of the Floreasca Emergency Clinical Hospital.

The first step of the surgery was performed by the neurosurgery team and consisted in a bicoronal incision followed by bifrontal trephination with the opening of the left frontal sinus. The purulent discharge was evacuated and the mucosa was stripped (head CT scan showed frontal sinusitis). In the right frontal sinus, an osseous, rough, tumoral mass, compressing the right frontal lobe and adhering to the dura mater in the superior region, was exposed (Figure 2).

Drilling was initiated from the posterior to the anterior wall of the frontal sinus in order to separate the nasal-ethmoidal-orbital fragment from the intracranial one. Drilling was continued towards the frontal sinus recess down to the right nasal cavity, where the orbital wall was drilled as to separate the osteoma from the lamina papyracea, which was enclosed by the tumor at this level (Figure 3).
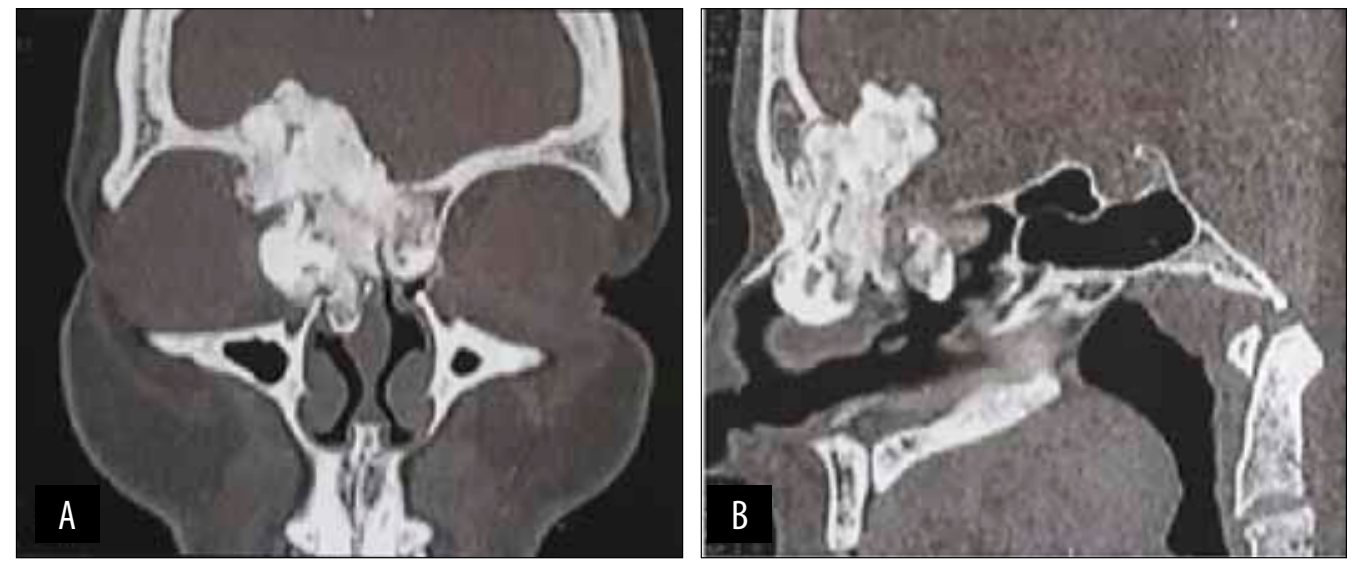

Figure $1 \mathrm{CT}$ imaging: giant frontal osteoma compressing the right frontal lobe (1A.- coronal view, 1B.- transversal view).

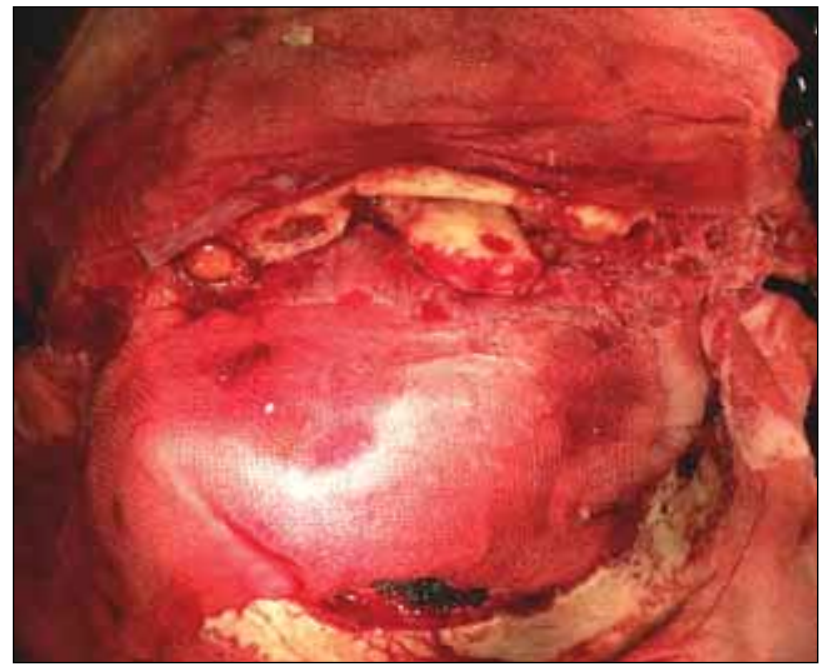

Figure 2 Frontal osteoma compressing the right frontal lobe.

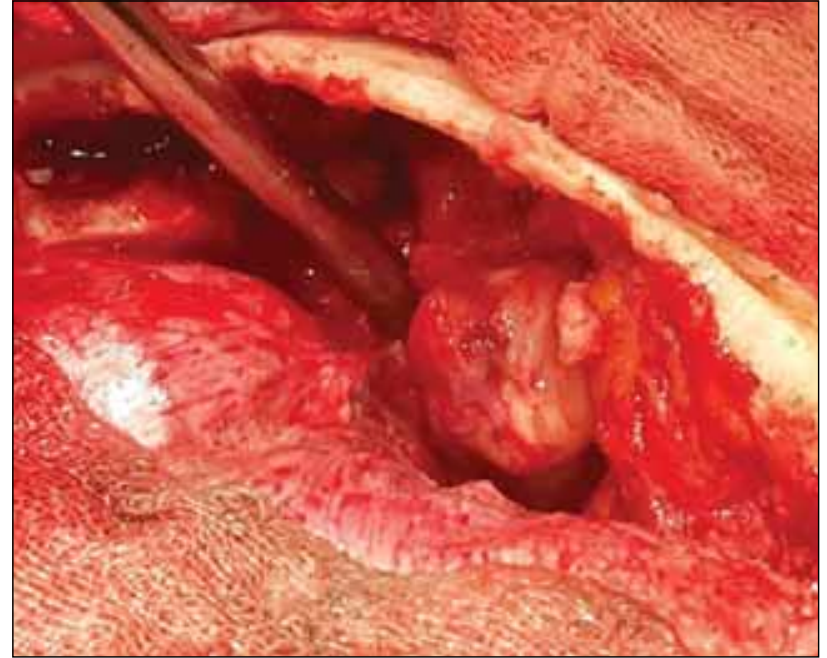

Figure 3 Ethmoidal orbital osteoma. 
At the anterior ethmoid level, after the dura mater detachment, the drilling was performed from the superior to the inferior part, at first on the right side and then extending to the posterior. In this manner, fragments of the osteoma were removed. On the left side, the drilling was realized in a similar way, but only in the anterior region. A small part of the lamina papyracea was removed with the left anterior ethmoid. The frontal sinuses were packed with special resorbable sponges.

Dural hemostasis was performed by electrocoagulation and no dural defect was detected. Reconstruction was performed using a bone graft (Figure 4).

An epicranial catheter was externalized through a lateral incision of the frontal skin. The anatomical layers were restored and the patient was bandaged. At the endoscopic examination through the right nasal cavity, a residual fragment of osteoma, located in the posterior ethmoid, was observed and extracted. A minimal CSF leak was noted in the posterior part of the frontal re-

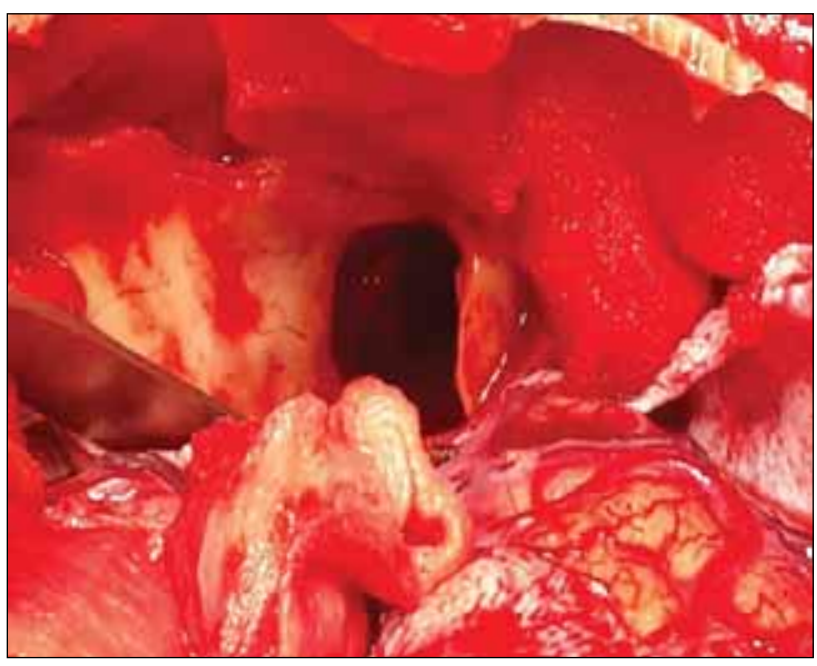

Figure 4 Cranial CT scan (coronal slice). Arrow showing the cribriform plate defect

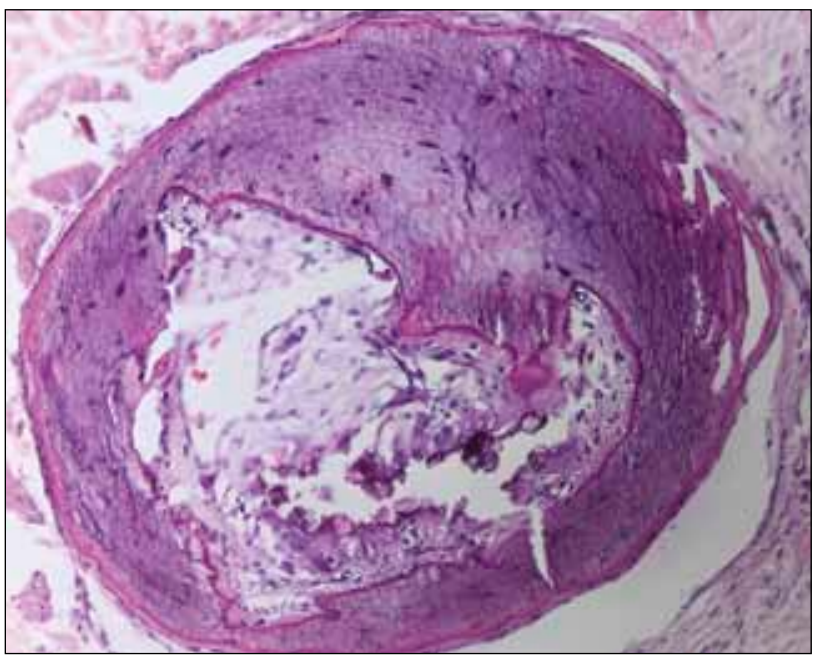

Figure 5 Sinusal osteoma: Hematoxylin \& eosin stain, 100x. cess, at the ethmoidal roof level, with a small dural defect (where dura mater was adherent to the tumor, difficult to dissect). A fragment from the middle turbinate (bone and mucosa) was placed at this level, then the middle turbinate was folded over the graft, Surgicel ${ }^{\circledR}$ and Nasopore ${ }^{\circledR}$ were applied, closing the fistula. Merocel® was used for the bilateral nasal packing.

Following surgery, the patient had a positive evolution; the nasal packing removal was performed after 24 hours and a minimal bleeding was present. The patient was hospitalized for the next six days in order to observe and treat any sign of CSF leak or endocranial complications. She was discharged from the hospital with complete surgical recovery and a good quality of life.

The tumoral mass resected during surgery was send to a histopathological exam and an immunohistochemistry investigation, which confirmed the diagnostic of rhinosinusal osteoma. The histopathologic examination revealed a broad bone trabeculae composed of dense, mature bone without atypical cells, with focal osteoblast rimming, dense calcification and some areas of hematopoietic marrow (Figure 5).

Sinusal osteomas are composed of large anastomosing trabeculae of mature bone, with non-atypical osteocytes and frequent osteoblastic rimming. Mitotic figures are rare. Stroma is scarce, fibrous, with minimal inflammatory infiltrate. Some osteoblastoma-like areas can be usually identified (narrow trabeculae of woven bone, also without atypia or mitotic activity), but no epithelioid osteoblasts can be found. Usually, the sinusal mucosa covers directly the lesion, without signs of invasion. Immunostaining for myeloperoxidase confirms the presence of bone marrow inside a pseudo-medullary space made out of anastomosing mature bone trabeculae (Figure 6)

The post-operative follow-up was decided at 1 month, 3 months and 6 months, and the nasal endoscopic examination did not show any sign of residual

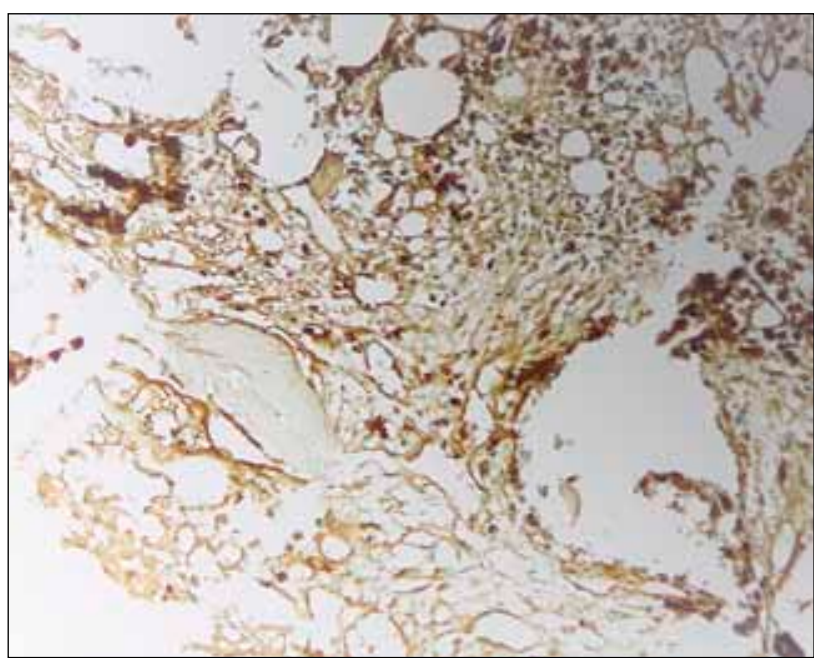

Figure 6 Immunostaining for myeloperoxidase, MPO stain 200x. 
or recurrent tumor or CSF leak. A CT-scan performed after one year showed complete removal of the tumor and the endoscopic exam with 0 and 70 degree endoscope revealed the integrity of the skull base with normal mucosa and no dural defect.

\section{DISCUSSIONS}

The available literature cites a low number of giant ethmoido-frontal osteomas ${ }^{3}$. Studies demonstrate that diagnosis following paranasal sinuses radiography has a frequency of $0.43 \%$ and up to $3 \%$ for computed tomography ${ }^{4}$. Tumors greater than $30 \mathrm{~mm}$ are considered giant osteomas 5 . The growing process is slow, between 0.44 and $6 \mathrm{~mm} /$ year, therefore the patient can be asymptomatic a long period of time ${ }^{6}$.

At present, the etiology is uncertain. There are numerous theories regarding the origin of these tumors: traumatic, infectious, embryologic ${ }^{7}$. Also, osteomas can be found in Gardner's syndrome, an autosomal dominant disorder (intestinal polyposis, cutaneous tumors, osteomas). For this reason, all patients suspected of an osteoma should undergo a colonoscopy (which we have recommended to our patient, with a negative result $)^{8}$. The gold standard of diagnosis is computed tomography. For asymptomatic, small-sized tumors, the temporization of the surgery is preferred with periodic imaging follow-up.

The differential diagnostic is made with fibrous dysplasia, ossifying fibroma, similar entities but with different clinical impact. Complications arise when the osteoma expands in the adjacent anatomical structures.

Even in the absence of important symptomatology, giant osteomas require surgical removal in order to prevent vicinity complications (orbital and intracranial). If orbital complications occur, the tumor must be gently removed, in order that all the orbital and ocular elements remain functional (periorbital region, extraocular muscles, optic nerve). If the tumor invades through the posterior wall of the frontal sinus, it must be elaborately resected, with the complete detachment from the dura mater, avoiding the possible intracranial complications (CSF leak). Even though the external approach would have permitted the closure of the CSF fistula found at the end of the surgery, we decided for an endoscopic self-grafted closure (middle turbinate). Another important surgical aspect was to repair the skull base, at ethmoidal level, with bone fragments separated from the surgical field. After the complete resection of the tumor, the remaining osseous defect at the level of lamina cribriforma was about $3 \mathrm{~cm}$ and it was necessary to close it with bony structures in order to avoid further complications (meningitis, meningocele, meningoencephalocele).

\section{CONCLUSIONS}

Giant rhinosinusal osteomas are rare tumors. The therapeutic aim is to completely remove the tumor using a combined (endoscopic and external) approach. Postsurgical morbidity varies with the type of surgery. Recurrence is a possibility if the tumor is not completely resected. The post-therapeutic follow-up is needed at 1 month, 3 months, 6 months and then annually.

The therapeutic success in giant osteomas with intracranial extension is granted by surgery with a multidisciplinary team, consisting of a neurosurgical team who ensures the tumor extraction from intracranial level and prevents specific complications, and an ENT team, whose critical part is to remove the sinonasal mass and to prevent orbital and ocular complications.

Last but not least, the endoscopic sinus surgical techniques are a real benefit in association with an external classic approach, thus increasing the quality of life of patients with giant complicated osteomas.

Conflict of interest: The authors have no conflict of interest.

Contribution of authors: All authors have equally contributed to this work.

\section{REFERENCES}

1. Smith M.E., Calcaterra T.C. - Frontal sinus osteoma. Ann Otol Rhinol Laryngol., 1989;98(11):896-900.

2. Budu V. - Chirurgia endoscopica rinosinusala. Editura Universitara "Carol Davila”, Bucuresti, 2014;p.69-70.

3. Izci Y. - Management of the large cranial osteoma: experience with 13 adult patients. Acta Neurochir (Wien), 2005;147(11):1151-1155; discussion 1155. Epub 2005 Aug 22.

4. Brunori A., de Santis S., Bruni P., Delitala A., Giuffre R., Chiappetta F. Life threatening intracranial complications of frontal sinus osteomas: report of two cases. Acta Neurochir (Wien), 1996;138(12):1426-1430.

5. Cheng K.J., Wang S.Q., Lin L. - Giant osteomas of the ethmoid and frontal sinuses: Clinical characteristics and review of the literature. Oncol Lett., 2013;5(5):1724-1730. doi: 10.3892/ol.2013.1239.

6. Koivunen P., Löppönen H., Fors A.P., Jokinen K. - The growth rate of osteomas of the paranasal sinuses. Clin Otolaryngol Allied Sci., 1997;22(2):111-114.

7. Vowles R.H., Bleach N.R. - Imaging case study of the month: frontoethmoidal osteoma. Ann Otol Rhinol Laryngol., 1999;108:522-524.

8. Alexander A.A., Patel A.A., Odland R. - Paranasal sinus osteomas and Gardner's syndrome. Ann Otol Rhinol Laryngol., 2007;116(9):658-662. 